\title{
Sém(h)antisme de la subjectivité essayistique: le cas de René Lapierre
}

\author{
Semantismo da subjetividade ensaística: o caso de René Lapierre
}

\author{
Emir Delic \\ Université Sainte-Anne - Canadá
}

\author{
$\diamond$
}

\begin{abstract}
Résumé: Si la pensée plurielle signifie souvent chemins inconnus et finalités incertaines, elle n'en est pas moins investie de propensions à certaines voies d'exploration et d'expression. Le but de cet article est de sonder l'une de ces voies, à savoir l'écriture essayistique. Un grand nombre de penseurs américains affichent une préférence marquée pour ce genre littéraire polymorphe, et ce, quels que puissent être les écarts historiques, culturels, politiques ou idéologiques qui les séparent. Comment expliquer cet attrait puissant de l'essai? Voilà la question à laquelle cet article proposera des éléments de réponse au moyen d'une étude des stratégies discursives de la subjectivité essayistique dont l'écriture de René Lapierre offrira une illustration emblématique.
\end{abstract}

Mots clefs: Essai; Genre littéraire; Théorie littéraire; René Lapierre

Resumo: Embora o pensamento plural frequentemente signifique caminhos desconhecidos e finalidades incertas, ele não deixa de ser propenso a algumas vias de exploração e expressão. O objetivo deste artigo é explorar uma dessas vias: a escrita ensaística. Um grande número de pensadores americanos demonstra uma clara preferência por esse gênero literário polimorfo, quaisquer que sejam as diferenças históricas, culturais, políticas ou ideológicas que os separam. Como explicar essa poderosa atração do ensaio? Eis a questão à qual este artigo proporá elementos de resposta por meio de um estudo das estratégias discursivas da subjetividade ensaística, da qual a escrita de René Lapierre será uma ilustração emblemática.

Palavras-chave: Ensaio; Gênero literário; Teoria literária; René Lapierre

Je réponds ordinairement, à ceux qui me demandent raison de mes voyages: que je sais bien ce que je fuis, mais non pas ce que je cherche.

Michel de Montaigne, Essais

Nombreux sont les gens de lettres des Amériques désireux de cerner les multiples spécificités de ces dernières, d'élucider les tensions qui les traversent, de démêler les contradictions et les apories qui les informent. Jacques Brault, Nicole Brossard, Herménégilde Chiasson, Joël Des Rosiers, Lise Gaboury-Diallo, Gabriel García Márquez, Pierre Nepveu, Octavio Paz, Dacry Ribeiro, Mordecai Richler, Domingo Faustino Sarmiento, Gerald Vizenor, autant d'auteurs dont les œuvres à la fois auscultent et construisent le vécu bigarré des populations américaines. Ce défi, ils le relèvent en recourant, implicitement ou explicitement, à une myriade de modes de "re/connaissance" (SIEMERLING, 2012) qui consistent à marier, à des degrés divers, des schèmes praxiques et gnosiques du Nouveau Monde à ceux de l'Ancien Monde. Pareils rapprochements entre les continents américain et européen, bien que susceptibles d'être tendancieux, ne sont certainement pas dénués d'intérêt. Il reste que les rapprochements entre les différentes cultures américaines s'imposent de plus en plus si l'on veut mieux comprendre tant leurs trajectoires individuelles respectives, majoritairement marquées par l'indétermination déstabilisante des logiques de 1' "entre-deux" (WALTER, 2003), que leurs relations mutuelles et dynamiques collectives, elles-mêmes inséparables de nos jours des "réseaux transculturels" 
mondiaux (IMBERT, 2014) qui se développent plus avant en se complexifiant. Aussi les Amériques se doivent-elles à l'heure actuelle de se penser au pluriel.

$\mathrm{Si}$ la pensée plurielle signifie souvent chemins inconnus et finalités incertaines, elle n'en est pas moins investie de propensions à certaines voies d'exploration et d'expression. Le but de cet article est de sonder l'une de ces voies, une voie qui touche à une pratique scripturaire révélatrice chez les penseurs américains et qui, si elle s'accuse en clair depuis le "virage transnational" (FOX, 2006) des études américaines il y a une quinzaine d'années, plonge ses racines bien au-delà du seuil du $\mathrm{XXI}^{\mathrm{e}}$ siècle. Il est question d'écriture essayistique. De fait, tous les auteurs mentionnés ci-dessus - et la liste dressée n'est aucunement exhaustive - ne font pas qu'une carrière littéraire et artistique; ils sont aussi des intellectuels qui cherchent de nouvelles façons de concevoir et de conceptualiser les sociétés qu'ils habitent, et qui tendent à formuler leurs réflexions sous forme d'essais. À vrai dire, un grand nombre de penseurs américains affichent une préférence marquée pour ce genre littéraire, et ce, quels que puissent être les écarts historiques, culturels, politiques ou idéologiques qui les séparent. Comment expliquer un tel attrait de l'essai? Voilà la question à laquelle cet article proposera des éléments de réponse au moyen d'une étude des stratégies discursives de la subjectivité essayistique dont l'écriture de René Lapierre offrira une illustration emblématique.

$$
* * *
$$

Professeur à l'Université du Québec à Montréal depuis 1981, René Lapierre porte plusieurs chapeaux littéraires. En font preuve ses publications parmi lesquelles on compte, outre des articles savants et des textes d'engagement, deux romans ${ }^{1}$, treize recueils de poésie $^{2}$ et sept essais ${ }^{3}$. Dans cette œuvre riche et variée, traversée tout entière par un souffle poétique, ce qui frappe, pour ce qui est de sa partie essayistique, ce sont les thématiques. L'entretien du désespoir (2001), Figures de l'abandon (2002), L'atelier vide (2003) et Renversements (2011), les quatre essais qui voient le jour au début du nouveau millénaire, se penchent sur des problématiques qui dépassent nettement les anciennes préoccupations québécoises axées sur l'affirmation culturelle et politique de la collectivité, et nourries abondamment du contexte de la Révolution tranquille des années 1960 et de celui du Référendum de $1980^{4}$. Ce changement d'optique se manifeste d'emblée dans $L^{\prime}$ 'entretien du désespoir ${ }^{5}$, essai sur lequel nous nous attarderons dans cette étude.

Délaissant les thématiques usuelles relatives à la langue et à l'identité québécoises, cette œuvre interroge la technoculture, les médias, la spectacularisation de la vie humaine et le système de marchandisation se déployant à l'échelle planétaire. Lapierre y jette la lumière sur l'envers des principes de l'individualisme et de la démocratisation des échanges caractéristiques de la postmodernité. Il montre plus précisément que ces principes, quoiqu'ils aient écarté certains métarécits oppresseurs de la modernité6, ne sont pas aussi libérateurs qu'on ne le croit, car, sous l'influence de l'élan capitaliste qui les sous-tend, ils contribuent à instaurer un monde régi par un tout autre principe: la mise à profit à tout prix. C'est, selon l'auteur, en raison de la prépondérance de ce principe capitaliste que l'être humain n'est devenu rien d'autre qu'un consommateur à exploiter et que s'installe progressivement une démoralisation généralisée qui le coupe des valeurs et des plaisirs simples, essentiels, non achetables, de la vie. Compte tenu de la portée globale de la problématique examinée dans $L$ 'entretien du désespoir, Lapierre, à l'instar de Jean-Pierre Issenhuth (2001) et de Pierre Ouellet (2003) par exemple, se signale ainsi comme faisant partie d'une nouvelle vague d'essayistes québécois qui, au lieu de limiter leur propos à la réalité québécoise, prennent celle-ci plutôt comme point de départ, comme ouverture sur le continent, sinon le monde entier.

Qu'on ne s'y trompe pas cependant: ce ne sont ni les problématiques abordées ni la généralisation du propos par-delà un contexte local, en l'occurrence québécois, qui font de Lapierre, ou de tout autre écrivain, un essayiste. Qu'est-ce, donc, un essayiste? La plupart des dictionnaires français, tel Le trésor de la langue française, indiquent simplement que c'est quelqu'un "qui écrit des essais". Cette définition laconique, apparemment banale, se complique toutefois aussitôt qu'on relance le questionnement en cherchant à savoir ce qu'est un essai. Car bien que, depuis son apparition sous la plume de Montaigne $^{7}$, l'essai ait connu une évolution certaine et

\footnotetext{
1 Comme des mannequins (1983) et L'été Rebecca (1985)

2 Profil de l'ombre (1983), Une encre sépia (1990), Effacement (1991), Là-bas c'est déjà demain (1994), Fais-moi mal Sarah (1996), Viendrastu avec moi? (1996), Love and Sorrow (1998), Piano (2001), L'eau de Kiev (2006), Traité de physique (2008), Aimée soit la honte (2010), Pour les désespérés seulement (2012) et La carte des feux (2015).

3 Les masques du récit (1980), L’imaginaire captif (1992 [1981]), Écrire l'Amérique (1995), L'entretien du désespoir (2001), Figures de l'abandon (2002), L'atelier vide (2003) et Renversements (2011).

4 Robert Vigneault résume finement l'emprise du nous collectif sur l'essayiste québécois: “[L]'essayiste québécois, sauf de rares exceptions, a eu pour ainsi dire la vocation non seulement de servir un propos, mais aussi un Pays (incertain, l'a-t-on assez répété) - service commandé, au Québec. Le je tend alors à se laisser occulter, voire avaler, par le nous d'une collectivité assez dévorante, semblerait-il, pour empêcher la libre expression du sujet individuel" (1994, p. 154). À ce sujet, voir également Mailhot (2005, p. 15-80).

5 Désormais, les références à cette œuvre seront indiquées par le signe $E D$, suivi du folio, et placées entre parenthèses dans le texte.

6 Songeons, par exemple, à l'emprise du pouvoir clérical ou à la place et au statut sociaux jadis réservés aux femmes.

7 Quoiqu'on convienne généralement que les Essais de Montaigne (1580) marquent la naissance du genre, d'aucuns soutiennent que celle-ci remonte à l'Antiquité. Georg Lukács, par exemple, tient Platon pour "le plus grand essayiste qui ait jamais existé et écrit" (1974, p. 27).
} 
qu'il ne constitue plus un genre mineur, il pose toujours un défi majeur de définition. En témoignent parfaitement les résonnances multiples de sa désignation, lesquelles Jean Starobinski a saisies avec acuité:

Essai [...] provient du bas latin exagium, la balance; essayer dérive d'exagiare qui signifie peser. Au voisinage de ce terme on trouve examen: anguille, languette sur le fléau de la balance, par suite, pesée examen, contrôle. Mais un autre sens d'examen désigne l'essaim d'abeilles, la nuée d'oiseaux. L'étymologie commune serait le verbe exigo, pousser dehors, chasser, puis exiger. Que de tentations si le sens nucléaire des mots d'aujourd'hui devait résulter de ce qu'ils ont signifié dans un passé lointain! L'essai autant dire la pesée exigeante, l'examen attentif, mais aussi l'essaim verbal dont on libère l'essor. (1985, p. 165)

Cette caractérisation tripartite de l'essai se répercute peu ou prou dans les usages variés qu'on en fait aujourd'hui. Qu'on le considère comme un "outil de recherche" (BELLEAU, 1986, p. 89), comme "un mode de connaissance, d'expérimentation de soi" (MARCEL, 1992, p. 324), comme un "écrit-qui-parle" (TREMBLAY, 2000, p. 1), ou encore comme une "forme d'art" (LUKÁCS, 1974, p. 13), les conceptions élaborées à son égard ne manquent pas et chacune d'elles se montre pertinente à sa manière. Peu importe cependant la définition avancée, l'essai semble toujours réussir à s'en dérober tout en en appelant d'autres. Loin de nous donc l'idée de vouloir trancher ici la question générique de ce genre éminemment polymorphe. Nous conviendrons au contraire avec René Audet que l'essai se présente sous plusieurs angles comme "une forme informe" (AUDET, 2005, p. 119). Or quand bien même il revêtirait des guises innombrables, l'essai possède, comme le souligne également Audet, des "mécanismes", des "rouages", des "moyens" de formulation de la pensée qui lui sont propres (AUDET, 2005, p.120). Si nous revenons maintenant à notre question initiale qui était de savoir ce qu'est un essayiste, nous pourrions, en tenant compte des constatations de Starobinski et d'Audet, y donner avec prudence la réponse suivante: est essayiste celui ou celle qui fait montre d'une maitrise de certaines stratégies énonciatives arrimées aux trois actes connotés par le vocable "essai", soit la pesée exigeante, l'examen attentif et l'essaim verbal. Les stratégies énonciatives qui retiendront notre attention dans la suite de cette étude se rapportent toutes à l'inscription complexe, dans le texte, du sujet de l'énonciation.

\section{La subjectivité essayistique: avatars et postures}

Que l'essai ait tendance à l'éclatement, cela ne surprendra guère quiconque a tant soit peu fréquenté ce type d'écrits, mais que dire du sujet de l'énonciation? Qui, au juste, parle dans un essai? Quelle est la démarche discursive de cette subjectivité qui tantôt s'y affiche, tantôt s'y efface? Et pourquoi prend-elle la parole? Malgré d'importantes études dans le domaine, notamment celles de Jean Marcel (1992) et de Robert Vigneault (1994), la question de la subjectivité essayistique demeure épineuse. Elle sera explorée ici à partir d'une étude de L'entretien $d u$ désespoir de René Lapierre et de l'hypothèse que, si l'on tient la subjectivité pour la pierre de touche de l'essai, c'est à condition qu'elle engage un véritable dialogue sur le sujet traité, dialogue qui implique une participation active du lecteur et qui sera idéalement poursuivi par ce dernier même après qu'il aura tourné la dernière page du texte.

Il est généralement admis que l'essayiste n'utilise pas la langue à des fins purement communicationnelles puisqu'il s'attache activement à la travailler. Alors qu'il se présente donc clairement comme un "écrivain", et non pas comme un "écrivant" (VIGNEAULT, 1994, p. 24), le "je" qui surgit à l'intérieur de l'essai et qui dénote en principe l'énonciateur qu'est l'essayiste occupe une place beaucoup plus ambiguë dans l'économie discursive. Les théoriciens ayant abordé la question ont généralement adopté deux perspectives différentes dont les principaux porte-parole sont Robert Vigneault, d'un côté, et Jean Marcel, de l'autre. Définissant l'essai comme un "discours argumenté d'un SUJET énonciateur qui interroge et s'approprie le vécu par et dans le langage" (1994, p. 21), Vigneault insiste sur le fait que le "SUJET énonciateur" est un "être de langage", un "JE de l'écriture" et non pas le "moi-je de l'auteur" (1994, p. 22). Marcel, en revanche, conçoit l'essai comme un "discours réflexif de type lyrique entretenu par un JE non métaphorique sur un objet culturel (au sens le plus large)" (1992, p. 318). Il va même plus loin: il édifie le "JE non métaphorique" en critère distinctif et décisif du genre, car, d'après lui, dès que le sujet énonciateur serait métaphorisé, il ne s'agirait plus d'un essai (1992, p. 316-317). Si, à première vue, ces deux perspectives ont l'air diamétralement opposées, elles ont le mérite d'être claires quand il est question de décider si un texte appartient au genre de l'essai. Car, qu'on adhère au point de vue de Vigneault ou à celui de Marcel, cette décision se résumerait à déterminer si le "je" du sujet de l'énonciation s'avère métaphorique ou non.

Il y aurait pourtant lieu de se demander si la présence d'un "je" métaphorique dans un texte exclut ipso facto celle d'un "je" non métaphorique. À plus forte raison qu'à $y$ regarder de près, même les deux théoriciens sèment du doute concernant une supposée position statique du "je". En ce sens, Vigneault, dans ses considérations sur l'œuvre de Pierre Vadeboncœur, déclare: "Franchi le pas: d'un je réel, hors texte, sujet prétendu de l'écriture, à la 
véritable écriture du je, fictive, littéraire, s'inaugure un jeu toujours inachevé, qui se renouvelle constamment par les métamorphoses du sujet de l'énonciation" (1994, p. 156). À ces propos de Vigneault, répondent en échos les remarques suivantes de Marcel:

Il arrive donc que dans certaines formes narratives (particulièrement le roman, plus rarement le conte ou la nouvelle), des fragments plus ou moins longs, isolables et détachables, répondent à la forme de l'essai telle que définie plus haut; une variation d'importance entre toutefois en jeu dans le cas du premier élément: le JE (non métaphorique dans l'essai stricto sensu) devient le JE métaphorique du narrateur (parfois considéré comme non métaphorique dans la mesure où il renvoie au $\mathrm{JE}$ réel de l'auteur - mais il n'y a pas lieu de soulever ici cette question difficile) ou, plus rarement, d'un personnage. (1992, p. 318-319)

De ces constatations éclairantes des deux critiques, ressort nettement la possibilité d'une rencontre entre les deux types de "je" qui paraissaient initialement contradictoires. En effet, pour complexe que soit la question du "je" essayistique, il nous semble que, comme le signale Élisabeth Nardout-Lafarge (2004, p. 32), dans tout essai s'actualise une dynamique entre les "je" métaphorique et non métaphorique. Pour s'en convaincre, on n'a qu'à considérer que, d'une part, quelques efforts qu'un auteur fasse pour décrire fidèlement son "moi" réel - c'est le cas, entre autres, dans le récit autobiographique -, aussitôt qu'il appose le "je" sur une page, ce "je", en intégrant le domaine de la narration, devient tant soit peu métaphorisé; d'autre part, étant donné que tout acte d'écriture s'inspire d'une manière ou d'une autre $\mathrm{du}$ vécu, un auteur projette nécessairement une part de soi dans un "je" narratif même s'il le souhaite non métaphorique. Envisagé dans cette perspective, le sujet de l'énonciation d'un essai ne saurait être statique, c'està-dire à jamais doté ou privé de métaphoricité. Tout porte à croire, au contraire, qu'il se constitue par une sorte de perpétuel vacillement entre les pôles métaphorique et non métaphorique sans toutefois jamais pouvoir élire domicile ni dans l'un ni dans l'autre. Voilà, soit dit en passant, la raison pour laquelle l'appellation de "subjectivité", dont nous usons dans ces pages, convient sans doute mieux que celle de "je" pour connoter la constitution fuyante du sujet de l'énonciation d'un essai.

Dans L'entretien du désespoir de René Lapierre, les différents positionnements de la subjectivité essayistique résultant de son va-et-vient entre les pôles métaphoriques et non métaphoriques se révèlent avec force dans les marques de l'énonciation ${ }^{8}$. Les plus évidentes de ces marques sont les pronoms personnels. Étonnamment, au début de L'entretien du désespoir, la subjectivité ne semble guère se manifester explicitement. Le fait que le pronom "je" n'y apparaît pas ne veut pas dire, toutefois, que la subjectivité en soit absente. C'est que, comme l'a fait valoir Catherine Kerbrat-Orecchioni (1980, p.32), même les textes qui se veulent les plus objectifs ou impersonnels contiennent des signes qui décèlent la griffe de l'énonciateur. Prenons, à titre d'exemple, le passage de suivant L'entretien du désespoir qui porte sur l'incitation continuelle adressée aux consommateurs (que nous sommes toutes et tous) et visant à les amener à capitaliser, dans toute l'acception du terme:

Savoir si réellement de telles possibilités [celles des promesses du marché économique] sont infinies n'importe bien sûr aucunement: dans les faits l'incitation à consommer se réitère tant et aussi longtemps que se maintient la possibilité d'un quelconque profit, toujours suffisant semble-t-il pour faire taire les scrupules. (ED, p. 22)

On investira, on fusionnera, on congédiera. ( $E D$, p. 22$)$

On achètera à la hâte, pour dix dollars, une camelote qui certainement ne vaut pas davantage, mais dont supposément le prix serait deux ou trois fois plus élevé ailleurs. On ne voudra pas savoir d'où viennent ce T-shirt, cette lampe, cette radiocassette. Rituellement s'exécute la dépense. Pas d'alternative, apparemment: on ne peut ni s'en passer, ni payer davantage. $(E D, \text { p. } 23)^{9}$

Ce passage illustre bien l'ingéniosité de la subjectivité qui cherche à s'effacer et qui déploie à cette fin plusieurs stratégies. La première de ces stratégies a trait à la modalité des verbes. On remarquera en ce sens l'emploi généreux de la voix passive, qui se traduit ici par les verbes pronominaux: "se réitère", "se maintient" et "s'exécute". À cette première stratégie s'ajoute l'emploi fréquent du pronom impersonnel "on". Porteur d'objectivité ou de neutralité, le “on" n'est pour autant jamais désincarné puisque sa signification est inévitablement tributaire du contexte de l'énonciation. Il importe de noter à ce sujet que, dans l'essai, ce contexte comprend aussi bien la diégèse, ou l'univers spatio-temporel du récit ${ }^{10}$, que le monde réel du lecteur. Sinon, il serait impossible de comprendre les diverses incarnations fluctuantes du pronom "on". Ainsi, dans la phrase "On investira, on fusionnera, on congédiera", le "on" désigne décidément

\footnotetext{
8 C'est-à-dire tous les éléments qui trahissent un locuteur dans un énoncé. Cf. Kerbrat-Orecchioni (1980).

9 L'entretien du désespoir étant construit de paragraphes fragmentés, nous les reproduisons comme tels dans nos citations. Nous indiquons toujours la page qui correspond à chaque paragraphe afin de rendre compte de cette fragmentation qui, on le verra, constitue un aspect déterminant de cette œuvre.

10 Selon la définition de Gérard Genette (1972).
} 
ceux qui tirent les ficelles de la machine capitaliste, à savoir les producteurs par opposition aux consommateurs. En revanche, c'est clairement à ces derniers que renvoie le "on" qui apparaît au dernier paragraphe de l'extrait: "On achètera à la hâte $[\ldots]$ une camelote $[\ldots]$. On ne voudra pas savoir d'où viennent ce T-shirt, cette lampe, cette radiocassette. [...] on ne peut ni s'en passer, ni payer davantage." Autre différence dans ces deux figurations du “on", la subjectivité essayistique s'exclut de la première (celle dénotant les producteurs) tandis qu'elle s'inclut dans la seconde (celle dénotant les consommateurs).

Ailleurs dans le texte, elle s'exprime également par l'intermédiaire du pronom "nous" et des adjectifs possessifs correspondants ("notre", "nos"). À l'instar de la seconde figuration du "on" dans l'extrait précédent, le "nous" réfère normalement à la masse des consommateurs:

L'extrême se présente, dans le langage promotionnel, comme le signe même de l'impossible mis à notre portée par le produit, réalisé par lui. $(E D$, p. 36)

Cet isolement [du désespoir créé par la marchandisation] [...] souligne que non seulement nous manquons toujours de quelque chose, mais que nous en manquons d'une manière particulière, inacceptable au regard de la consommation standardisée. (ED, p. 41)

Même si les pronoms "on" et "nous" peuvent alors tous les deux renvoyer à la subjectivité essayistique, il existe une différence non négligeable dans leur usage respectif. Attendu que le lecteur, par le statut de consommateur qu'il partage avec l'essayiste, associera intuitivement le pronom "nous" au groupe des consommateurs, il n'est pas nécessaire de corser le contexte de l'énonciation pour clarifier la signification du "nous". Il n'en va pas du tout de même pour l'usage du pronom "on". Car pour établir une distinction claire et nette entre consommateurs et producteurs au moyen de ce pronom et pour signaler son appartenance à l'un ou l'autre groupe, la subjectivité essayistique est tenue d'expliciter le contexte de l'énonciation. Revenons à cet égard à un passage déjà cité et notons-y comment l'emploi de compléments circonstanciels adverbiaux se greffe à la mention d'exemples spécifiques d'habitudes de consommation dans le but de mettre en relief que le "on" représente le consommateur exploité et que la subjectivité s'identifie à lui:

On achètera à la hâte, pour dix dollars, une camelote qui certainement ne vaut pas davantage, mais dont supposément le prix serait deux ou trois fois plus élevé ailleurs. On ne voudra pas savoir d'où viennent ce T-shirt, cette lampe, cette radiocassette. Rituellement s'exécute la dépense. Pas s'alternative, apparemment: on ne peut ni s'en passer, ni payer d'avantage. ( $E D$, p. 23; nous soulignons).

Tout bien considéré, les pronoms "on" et "nous" représentent donc deux figurations, deux avatars, dont se sert la subjectivité essayistique afin d'éviter de se manifester sous la forme d'un "je" explicite.

L'effet que peut produire un tel effacement du sujet de l'énonciation est double. D'un côté, on peut y voir une manière de faire valoir, voire de faire accepter à autrui, une vérité établie, l'usage des pronoms "on" et "nous" insufflant au discours des allures objectives et sincères (ANGENOT, 1982, p. 49); d'un autre côté, l'effacement des marques de l'énonciation peut aussi permettre au lecteur de peser le discours de la subjectivité essayistique plus aisément, car il ne le reçoit pas par l'entremise d'un sujet énonciateur explicite (VANDENDORPE, 2004, p.136-137). C'est ce deuxième effet de l'effacement de l'énonciation, grâce auquel le lecteur joue un plus grand rôle sur "la scène énonciative ${ }^{11}$ ", qui est à l'œuvre dans L'entretien $d u$ désespoir. Le confirme l'usage stratégique, dans tout l'essai, de la première personne du singulier. N'apparaissant que dans quatre passages du texte ${ }^{12}$, elle renvoie, à une exception près, non pas à un "je" ressortissant à l'essayiste, mais à un "je" ressortissant à tout consommateur. Tout comme dans le cas du pronom "on", la signification de ce "je" à caractère universel se fonde sur plusieurs composantes du contexte de l'énonciation (d'où la longueur du passage ci-après):

Il semble de plus en plus évident que dans cette entreprise de fascination/dévoration l'humain soit devenu tributaire du non-humain - du quantitatif, du numérique - et ne puisse être envisagé qu'à cette condition. [...] En termes d'intéressement, entre le réel et le virtuel, le rapport se construit toujours au détriment de la réalité. $(E D$, p. 49$)$

Parce que la réalité, en regard des pratiques d'accélération du rendement et d'accroissement du profit, est pauvre, insuffisante, irrémédiablement lente. Et que dans cette lenteur elle est inattentive non seulement à ce que je suis, dont elle n'a nul souci, mais encore à ce dont je suis séparé. Arbres et nuages, feuilles, terre, choses effacées, impondérables, recommencements. ( $E D$, p. 50 ; nous soulignons)

Dans sa lenteur irrecevable, elle me ramène constamment à ce que je ne crois pas être, dont

\footnotetext{
${ }^{11}$ L'expression est de Robert Dion, Anne-Marie Clément et Simon Fournier (2000, p. 27)

12 Voir $E D$, p. 33, 50, 51, 87. Nous faisons abstraction ici de l'usage de la première personne dans les citations d'annonce publicitaire qui ponctuent le texte.
} 
elle me dit cependant constitué. Arbres et nuages, étoiles, pierres. Et ce faisant traite avec exactitude, sans douceur, mais sans violence, mon espoir de la surmonter. À cet espoir elle dit détrompe-toi, renonce. Mais tout autour ça dit autrement, dépêche-toi, exécute-toi, les mots mêmes dont s'enivre l'entretien du désespoir. (ED, p. 51; nous soulignons)

Compte tenu du contexte global établi au premier paragraphe de cet extrait qui traite des lourdes conséquences de la marchandisation, il ne fait pas de doute que le pronom "je" énoncé par la suite est assimilable à tout consommateur. La subjectivité essayistique invite ainsi le lecteur à s'identifier à ce "je" pour lui faire voir et sentir sa condition "humain[e]" ou bien "non humain[e]" de "sujet privé" (ED, p. 87), un sujet aux prises avec l'implacable instrumentalisation attenante aux "pratiques d'accélération du rendement et d'accroissement du profit" (ED, p. 50). Remarquons en outre que l'emploi de l'impératif dans cet extrait n'est pas fortuit puisqu'il sert à souligner que le consommateur ne se trouve pas à l'origine du discours. En effet, qu'il soit à la deuxième personne du singulier ("tu") ou du pluriel ("vous") - comme c'est le cas ailleurs dans l'essai -, l'impératif remplit toujours la même fonction dans L'entretien du désespoir: mimer les divers discours idéologiques interpellant le lecteur au nom de telle ou telle cause. Intimement lié à l'utilisation des pronoms "je" et "nous" en tant que figurations du consommateur, l'impératif constitue, en ce sens, une autre stratégie dont dispose la subjectivité essayistique afin d'aménager la scène énonciative.

Si l'on envisage l'ensemble des marques de l'énonciation de la subjectivité essayistique que nous venons de repérer dans une optique globale et schématique, on pourrait affirmer qu'elles se présentent sous un mode effacé au début et à la fin de L'entretien du désespoir alors qu'en son milieu, elles s'avèrent plus accusées. Cette distribution des marques de l'énonciation a pour résultat une sorte d'effet "cône" qui informe la structure globale de l'essai. Considéré ainsi, celui-ci s'ouvre et se clôt sous le signe d'un regard global sur le système de marchandisation alors qu'au cœur de l'œuvre, comme au cœur de la problématique de l'essai, se loge l'individu et l'évocation des conséquences particulières qu'entraîne, sur lui et ses semblables, l'omniprésence et l'apparente omnipotence de la logique de la mise à profit à tout prix. Cet effet "cône" se confirme au demeurant par le fait qu'en plus du “je” universel déjà évoqué, l'œuvre abrite, au plus creux du parcours de la subjectivité essayistique, un " $\mathrm{je}$ " qui renvoie véritablement à cette dernière et qui ne se manifeste qu'une seule fois ${ }^{13}$.

Que L'entretien $d u$ désespoir décrive ainsi une trajectoire discursive déterminée démontre non seulement la capacité de la subjectivité essayistique à adopter différents avatars, mais encore son habileté à prendre différentes postures discursives vis-à-vis d'une multitude d'intervenants sur la scène énonciative. Il s'ensuit que, que sa présence soit effacée ou accusée, et quel que soit son avatar pronominal ("on", "nous", "tu”, "vous", “je”), la subjectivité essayistique fait immanquablement fonction de gérante de l'énonciation en ce qu'elle s'attache à polariser les actes de discours qui s'y actualisent. Il lui est impossible, en effet, d'aménager la scène énonciative sans contraster les discours de plusieurs acteurs les uns avec les autres et sans se positionner à leur égard en signalant ses alliés et ses adversaires (DION, CLÉMENT et FOURNIER, 2000, p.36). De cela aussi, L'entretien $d u$ désespoir nous offre une excellente illustration. Car, dans cet essai, la subjectivité ne se contente pas de se jouer d'avatars pronominaux; elle cite aussi nombre d'auteurs, d'œuvres et de slogans publicitaires ${ }^{14}$. Tout se passe toutefois comme s'il s'agissait moins de céder la parole à telle personne, à tel personnage ou à tel "message" que de parler tantôt à travers, tantôt avec, tantôt contre eux, et d'éprouver de la sorte des idées avancées. Voilà qui nous amène à une exploration de la démarche discursive qui s'active dans l'essai.

\section{Démarche fragmentaire et approche dialogique}

L'aptitude de l'essai à récuser les binarismes est sans l'ombre d'un doute sa qualité primordiale, celle qui lui confère une inestimable valeur heuristique (VIGNEAULT, 1994, p. 291-295). Or cette même aptitude ne ferait-elle pas aussi obstacle à ce genre polymorphe, qui s'est déjà vu qualifier de "biographie, mais sans événements" (MARCEL, 1992, p.325) et de "science, moins la preuve explicite" (ORTEGA Y GASSET, 1936, p. $\left.9^{15}\right)$ ? Sans doute, puisque la plupart des caractérisations paradoxales de l'essai reposent sur l'opposition épineuse entre art et science ${ }^{16}$. Mais cette opposition binaire est-elle insurmontable? Cela semble moins certain. Car, pareil à la subjectivité essayistique qui transcende l'opposition entre les "je" métaphorique et non métaphorique, l'essai en tant que genre se montre apte, à bien des égards, à puiser à la

\footnotetext{
$\overline{13}$ Nous reviendrons plus loin à ce passage clef.

${ }^{14}$ Sont cités, entre autres, Poly Styrene du groupe X-Ray Spex (ED, p. 24), Guy Debord (ED, p.41), Giacomo Leopardi (ED, p.66) et Charles Baudelaire $(E D$, p. 75$)$ de même que les publicités de Bell Sympatico (ED, p. 16), Apple Computers (ED, p. 19), Parfums Givenchy (ED, p. 53) et Toyota (ED, p. 76).

15 Cité par Marcel (1992, p. 324).

${ }^{16} \mathrm{C}$ 'est de là que procède, au fond, la question de savoir si les monographies et plus généralement les traités de type scientifique sont à ranger sous la catégorie de l'essai. Lukács est, à notre connaissance, le premier théoricien du genre à établir une distinction entre les "véritables essais" et "ces écrits, utiles, mais qualifiés improprement d'essais, qui ne nous donneront jamais plus qu'un enseignement, des données et des “contextes"” (1974, p. 13).
} 
fois dans l'art et dans la science. Et cela, même s'il se nourrit davantage, à nos yeux, du champ artistique. Cette hypothèse trouve un renfort si l'on considère la facture de la démarche discursive de la subjectivité essayistique.

Précisons-le d'entrée de jeu: l'essai ne saurait prétendre à l'objectivité propre aux sciences pures, ne serait-ce que parce que celles-ci s'évertuent de faire du langage rien d'autre qu'un moyen de communication évadé autant que possible de toute marque susceptible de renvoyer à la griffe personnelle de l'énonciateur. Cette opposition entre discours objectif et discours subjectif, qui a encore cours dans la pensée contemporaine, est intimement liée à l'opposition entre la linéarité du discours scientifique et la démarche non linéaire de l'essai. Theodor Adorno souligne à ce sujet:

[L'essai] réfléchit sur ce qu'il aime et ce qu'il hait, au lieu de présenter l'esprit comme une création ex nihilo, sur le modèle de la morale du travail illimitée. [...] Il ne remonte pas à Adam et Ève, mais part de ce dont il veut parler; il dit ce que cela lui inspire, s'interrompt quand il sent qu'il n'a plus rien à dire, et non pas quand il a complètement épuisé le sujet: c'est pourquoi il se range dans la catégorie des amusettes. (2003, p. 51)

L'essai se présente alors comme un lieu de réflexion où des idées apparaissent au hasard des chemins et des rencontres, où elles mijotent, où de nouvelles idées s'ajoutent au gré de la réflexion. Ce cheminement aléatoire de la pensée qui empêche de prétendre à l'exhaustivité $\mathrm{du}$ discours scientifique est effectivement l'une des premières caractéristiques qu'on a reconnues à l'écriture essayistique. Ainsi lit-on dans l'Encyclopédie de Diderot et d'Alembert que l'essai désigne:

[...] des ouvrages dans lesquels l'auteur traite ou effleure différens sujets, tels que les essais de Montaigne, ou des ouvrages dans lesquels l'auteur traite un sujet particulier, mais sans prétendre l'approfondir, ni l'épuiser, ni enfin le traiter en forme $\&$ avec tout le détail \& toute la discussion que la matiere peut exiger $[\mathrm{sic}] .(1751, \mathrm{p} .982)$

Si elle ne prétend pas épuiser à fond un sujet, si elle ne se conforme pas à la démarche des sciences pures, bref, si sa démarche est "méthodiquement non méthodique" (ADORNO, 2003, p.66), la subjectivité essayistique possède néanmoins une démarche discursive. Quelle est cette démarche? Celle qu'Aristote appelle l'enthymème ${ }^{17}$. Il s'agit d'une forme de raisonnement par déduction qui contraste avec le syllogisme dialectique sous deux aspects majeurs. D'abord, au regard d'une démarche scientifique,

\footnotetext{
17 Voir Aristote (2014, II, xxvii, 70 a). Nous reprenons ici le rapprochement entre l'enthymème et la discursivité de l'essai établi par Terrasse (1977) et Vigneault (1994).
}

la valeur des preuves intrinsèques, qui sont censées "établir les faits", n'est pas la même dans le discours enthymématique (TERRASSE, 1977, p.133). Au lieu de la démarche purement logique du syllogisme pensons à la chaîne "majeure, mineure, conclusion" - la démarche du discours enthymématique est plus souple en ce qu'elle s'appuie sur des probabilités, des vérités admises et des opinions générales (VIGNEAULT, 1994, p. 28). Ensuite, les preuves extrinsèques, c'est-à-dire ceux qui travaillent l'ethos et le pathos en vue de persuader l'énonciataire, occupent une place de premier plan dans l'enthymème qui valorise divers procédés rhétoriques. Cette valorisation de la rhétorique, couplée à la démarche foncièrement intuitive de la subjectivité essayistique, fait en sorte que, dans l'essai, s'accumulent et s'enchevêtrent exemples, anecdotes, citations et témoignages, tous scandés de constatations d'ordre moral et philosophique (VIGNEAULT, 1994, p. 28). C'est très précisément cet amalgame plus ou moins fortuit d'éléments hétéroclites qui engendre la nature "zigzagante" (ANGENOT, 1982, p. 57) ou "décousu[e]" (TERRASSE, 1977, p. 136) de la démarche discursive de la subjectivité essayistique. Cela dit, examinons maintenant la manière dont cette démarche discursive se décline dans $L$ 'entretien du désespoir?

Construit d'une sorte de fragments de réflexion qui se présentent sous la forme de paragraphes détachés et qui sont rassemblés de façon à former sept "chapitres", L'entretien du désespoir s'érige d'emblée sous le signe de ruptures. Il va sans dire que la discontinuité dérivant de cette parcellisation de l'œuvre s'amplifie aussi bien par les avatars et les postures de la subjectivité essayistique déjà constatés que par les procédés proprement rhétoriques mis en mouvement dans la quasi-totalité des fragments qui composent le texte. En voici un exemple patent:

Appareils audio, vins et restos, mode, voyages, événements sportifs, un coup d'œil aux rubriques quotidiennes des journaux suffit pour reconnaître les pratiques qui sont valorisées par l'industrie de la distinction, et pour mesurer l'ampleur de la dépendance qui se fonde sur elles. Et sur l'admiration de l'argent dont les fabriques de millionnaires instantanés (informatique et casinos, jeux télévisés, success stories de la finance et du showbiz) sont la caricature. $(E D$, p. 36)

Cet extrait s'avère intéressant sous deux points de vue. Remarquons, pour commencer, l'accumulation d'exemples qui, relevant de domaines divers, permettent à tout consommateur de s'y reconnaître puisqu'ils servent de preuves censées appuyer l'affirmation, faite plus tôt dans le texte, relativement à la dépendance développée par les "sujets" perpétuellement "privés". Ce court extrait met également en relief la manière dont la subjectivité 
essayistique élabore un véritable champ sémantique autour de la marchandisation et de ses failles: "industrie", "dépendance", "argent", "fabriquer", "millionnaires instantanés", "showbiz", l'usage de tous ces termes n'est en rien gratuit. Le champ sémantique qui se profile de la sorte à l'intérieur de ce fragment de réflexion n'est pas sans rappeler d'autres fragments. Loin de là. À preuve ces passages qu'une vingtaine de pages séparent de l'extrait précédent:

Dans le lexique du profit, la rapacité fait réellement de la vie la prédatrice de la vie, et fonde avec aplomb son enthousiasme sur une absolue positivisation du rapport à l'existence, uniquement régie par du consommer et du produire, et allergique à tout différement [sic]. "Attendre ça rend fou" - Bell Sympatico. (ED, p. 16)

Cette règle d'immédiateté s'insurge contre la lenteur naturelle (les discontinuités et les résistances) des processus et, pressée du résultat, stimule une productivité compulsive dont elle fait l'éloge en termes de rendement. Elle a en horreur tout contretemps, qu'elle interprète comme temps mort, et cherche à triompher des processus - alternances de cycles, travail/repos, mouvement/arrêt - en les purifiant de toute intermittence pour les reformuler en quelque chose de continu, non-stop, 7/7, 24/24. (ED, p. 17)

Ce faisant elle ne peut que traduire dans une hâte toujours plus grande, et dans un temps de plus en plus compté, le rapport à la vie - c'est-à-dire aux objets qui sont devenus dans l'entretien du désespoir les signes vides de la vie. $(E D$, p. 17)

Le champ sémantique du "lexique du profit" se déploie ici de toutes ses forces. "Consommer", "produire", "immédiateté", "rendement”, "non-stop", "7/7", "24/24", un terme en chasse un autre pour que le tout aboutisse au "signes vides de la vie". Comment un consommateur ne se reconnaîtrait-il pas dans ces "processus", d'autant que dans L'entretien du désespoir s'insinuent périodiquement des slogans publicitaires particulièrement incisifs, tel "Attendre ça rend fou ${ }^{18 "}$ ? Par ailleurs, cette immédiateté, ce non-stop qui mènent au désenchantement des rapports de l'être humain à l'égard de sa vie ne sont pas sans parenté aux "pratiques [...] valorisées par l'industrie de la distinction" et les "fabriques de millionnaires instantanés" (ED, p. 36), qui sont mentionnées dans le premier fragment de réflexion cité ci-dessus. De fait, les échos qui vibrent entre les fragments que nous venons de mentionner se prolongent sur l'ensemble de L'entretien du désespoir. Autrement dit, tous les fragments de réflexion qui constituent le vaste champ thématique de "l'entretien du désespoir" que défriche cet essai de Lapierre se répondent,

\footnotetext{
$\overline{{ }^{18} \mathrm{Au} \text { total, vingt-deux }}$ annonces publicitaires sont citées dans L'entretien du désespoir.
}

se répercutent, s'enrichissent de manières plus ou moins accentuées, et ce, indépendamment de la longueur des fragments. Que l'on songe ici, par exemple, au titre du premier chapitre intitulé "Promesses" qui aborde les promesses vides créées par le marché de consommation. À la fin du même chapitre, les "Promesses" du titre ne peuvent que réverbérer, sur un ton plaintif, voire douloureux, sur les deux fragments finals:

À ce point devient lisible l'effrayante totalité du désespoir, le caractère systématique et ordonné de son entretien. Au-delà, plus rien. (ED, p. 26)

Ni mot, ni geste, ni ciel. Une flaque de mort. (ED, p. 26)

L'établissement de telles connexions et correspondances thématiques plus ou moins ténues montre à quel point la subjectivité essayistique dans L'entretien du désespoir ne vise ni l'exhaustivité ni la systématicité. Élaborant une réflexion par associations intuitives d'idées, elle parle moins des problématiques de marchandisation et de démoralisation qu'autour d'elles. Il n'empêche que cette démarche de nature enthymématique demeure tout à fait valable, d'autant plus que le fait d'accumuler et d'associer les fragments de réflexion, plutôt que de les enchaîner suivant un principe syllogistique, permet à la subjectivité essayistique et au lecteur d'envisager les questions abordées sous des angles multiples et variées. À vrai dire, la subjectivité essayistique dans cet essai de Lapierre est tout à fait consciente du grand avantage de son approche approximative des questions traitées: "Changeons un peu de direction", proclame la subjectivité essayistique au début du chapitre "Une joie sans nom" (ED, p. 59). Or, si elle peut facilement changer de direction et inciter le lecteur à le faire avec elle, c'est parce que son esprit est en ébullition et qu'elle ne se garde pas de s'aventurer sur les chemins inopinés. Aussi la démarche enthymématique de la subjectivité essayistique, loin de limiter le propos, donne-t-elle libre cours à toutes sortes de pistes de réflexion. "J'ajoute, mais je ne corrige pas", c'est bel et bien cette sage leçon de Montaigne (1998, p. 276) qu'elle met à profit.

De plus, même si la subjectivité essayistique parle moins d'une problématique qu'autour d'elle, et même si sa réflexion se développe plus circulairement que linéairement, il ne faudrait pas en conclure qu'elle n'avance pas. Car, bien que dans tout essai se déchaînent les forces tant centrifuges que centripètes, il reste que les dernières l'emportent sur les premières et que la pensée chemine. Dans L'entretien $d u$ désespoir, ce patient cheminement de la pensée se signale globalement par les intertitres qui, pareils aux courbes d'une spirale ascendante, représentent les étapes discursives, sinon cognitives ${ }^{19}$. Les voici dans leur ordre séquentiel: 
"Promesses", "Cellulaire", "En revenir", "Une joie sans nom", "Portrait de Daisy Buchanan", "Forfaits" et "Péremption". Comme en témoigne l'enchaînement de ces titres, la réflexion de la subjectivité essayistique avance, mais elle le fait de manière discontinue, aléatoire, souvent surprenante, précisément parce qu'elle se fonde sur l'association de divers fragments qui composent l'essai. À y regarder de près, L'entretien du désespoir fournit en définitive un excellent exemple d'un essai constitué par ce que Christian Vandendorpe appelle des fragments de type "éclats", puisque les divers fragments de réflexion de cet essai peuvent être considérés comme "des 'éclats' détachés d'un même diamant" (2004, p. 132). Et il va sans dire que cette composition fragmentaire s'harmonise parfaitement avec la démarche foncièrement dialogique de la subjectivité essayistique.

\section{En quête de sens: lyrisme et sém(h)antisme}

Si L'entretien $d u$ désespoir prend appui sur les réflexions et réfractions de divers fragments "éclats", ces réflexions et réfractions ne s'activent pas seulement entre les mots et leurs significations. Elles s'activent aussi, et de manière encore plus prégnante, entre les sons, les sonorités, les rythmes de cet essai, éléments proprement littéraires inséparables du lyrisme et de la poésie.

On aura certainement remarqué, à la lecture des extraits déjà cités ci-dessus, le souffle lyrique qui traverse L'entretien du désespoir. On en serait d'ailleurs arrivé au même constat si cette analyse s'était basée sur n'importe quel autre essai de René Lapierre. Ce dernier ne fait cependant pas figure d'exception à cet égard, dans la mesure où le lyrisme constitue, à l'instar de la subjectivité avec laquelle il va de pair, une caractéristique saillante du genre de l'essai, imprégné qu'il est toujours d'une certaine tonalité poétique ${ }^{20}$. Ce n'est effectivement pas un hasard si l'essai se voit souvent rapproché de la poésie: Georg Lukács, par exemple, considère celle-ci comme "sa sœur" (1974, p. 27); Wil Schlegel tient l'essai pour un "poème intellectuel21"; et Robert Vigneault perçoit l'essai tel que pratiqué par Pierre Vadeboncœur comme un "poème idéel" (1994, p. 143). À la lueur de telles constatations répétées, relevant de différents objets d'études et de différents contextes, il serait difficile de nier la tonalité poétique de l'essai. Mais à quoi tient-elle? Et qu'elles en sont les retombées?

À bien y penser, la tonalité poétique du genre essayistique est reliée au but premier de l'essayiste: la description de la réalité 22 . Plusieurs théoriciens du genre le confirment. Jean Marcel souligne à cet effet que l'objectif de l'essayiste consiste en une appréhension intime du vécu qui s'effectue sur un mode lyrique. Vigneault, pour sa part, qualifie cette expérience particulière qu'éprouve l'essayiste de "dynamique événement de l'âme" (1994, p. $65^{23}$ ) et c'est sans doute cette même expérience qu'évoque Montaigne quand il se demande: "Et quand serai-je à bout de représenter une continuelle agitation et mutation de mes pensées, en quelque matière qu'elles tombent [...]?” (1998, p. 249). De là nous pouvons tirer deux conclusions: primo, un (véritable) essayiste, face au monde qui l'entoure ou dont il fait partie, pèse et soupèse le réel, le filtre en quelque sorte au crible de sa conscience individuelle pour en saisir les essences; secundo, le saisissement de ce "dynamique événement de l'âme" s'effectue sur un mode lyrique.

Aussi fuyant et abstrait que ce "dynamique événement" paraisse, René Lapierre semble appartenir à cette famille d'essayistes qui l'éprouvent. Non seulement ses écrits en font preuve, mais encore l'extrait suivant tiré de la quatrième de couverture de Là-bas c'est déjà demain (1994) met en lumière la part "intime" de sa démarche artistique:

Les textes de ce recueil proposent une poésie intensément narrative, aussi détachée que possible de la tentation lyrique du moi. Ce livre évoque par là le travail de la photo: ce regard décentré de soi qui pourtant renvoie à un sens intime des êtres et des choses, c'est-à-dire à ce point particulier de l'émotion qui se trouve à la limite de nos forces, et parfois même au-delà.

Faire voir, entendre et sentir un "sens intime des êtres et des choses" est selon nous précisément le dessein de René Lapierre et, par voie de conséquence, celui de la subjectivité essayistique dans ses essais, y compris L'entretien du désespoir. La question se pose dès lors de savoir comment ce dessein se réalise.

\footnotetext{
${ }^{19}$ Montaigne précise à ce propos: “[...] [J]e me donne loi d'y attacher, comme ce n'est qu'une marqueterie mal jointe, quelque emblème [incrustation] supernuméraire. Ce ne sont que surpoids, qui ne condamnent point la première forme, mais donnent quelque prix particulier à chacune des suivantes par une subtilité ambitieuse. Et là toutefois il adviendra facilement qu'il s'y mêle quelque transportation de chronologie" (1998, p. 276)

${ }^{20} \mathrm{Il}$ est à remarquer que Charles E. Whitmore associe l'essai, non pas à une forme, mais à un ton ("not a form, but a tone") (1921, p. 557).

21 Cité par Marcel (1992, p. 317).

22 Jean Terrasse rappelle à juste titre que cet objectif constitue l'une des trois visées de la rhétorique, les deux autres ayant trait à l'ethos (édifier l'image de l'énonciateur) et au pathos (émouvoir l'énonciataire) (1977, p. 128).

23 Vigneault s'inspire ici de Lukács qui, quant à lui, note que cette expérience particulière consiste en une "conception du monde dans sa pureté non voilée comme événement de l'âme, comme force motrice de la vie" (1974 [1911], p. 19). Et il ajoute: “[L]es expériences vécues pour l'expression desquelles sont nés les écrits des essayistes ne deviennent conscientes chez la plupart des gens que par la contemplation des tableaux ou la lecture des poèmes" (22)
} 
Le premier élément de réponse à cette question concerne la démarche enthymématique déjà interrogée. Il suffira donc de noter ici que la subjectivité fait fi de la démarche syllogistique justement parce que la réalité, qui lui fournit en quelque sorte les matériaux de sa réflexion, est foncièrement décousue et intermittente. Ensuite, si l'objectif de la subjectivité essayistique est l'appréhension intime du vécu et qu'elle finisse par acquérir une vision contestataire de la réalité, cela ne signifie pas qu'elle nie au préalable toute vérité admise, fût-ce parce que cela relèverait d'une démarche syllogistique prônant toujours un principe de base. En effet, c'est plutôt en contemplant la réalité telle quelle, dans son état brut, que la subjectivité essayistique dans $L$ 'entretien $d u$ désespoir réussit à l'éclairer sous un jour nouveau. Considérons, à titre d'exemple, les passages suivants qui confrontent deux visions divergentes de la réalité:

Il est vrai qu'au gré de la modification des rapports économiques et sociaux notre sens du nécessaire se modifie profondément, et comporte des objets de plus en plus variés. Il n'en reste pas moins que la promotion de la nécessité qui dans ce contexte donne bonne conscience à la surconsommation constitue une très profonde contradiction dans les termes. (ED, p. 44)

Il y aurait lieu d'en rire si elle ne se fondait pas sur l'injustice, et n'œuvrait à créer à même le manque (dans toutes ses variantes et jusqu'aux formes les plus dures de l'indigence) la suggestion d'un bonheur dans lequel la "récompense" (ne vous privez pas plus longtemps) prend la forme de l'asservissement, ou plutôt le renforce en feignant de le contrer (rien à payer avant juin 2003). (ED, p.44)

Si cet ensemble de fragments de réflexion s'ouvre sur une concession relativement à la circulation des biens et des êtres ${ }^{24}$ promise par la démocratisation des échanges, ce n'est que pour mieux en éclairer par la suite les conséquences morales. En outre, on remarquera dans le deuxième passage l'emploi d'incitations de type publicitaire fort répandues de nos jours ainsi que la signification altérée, ou bien dénaturée, du mot "récompense", qui signifie "asservissement". Le renversement de signification que subit ici le terme de "récompense" illustre bien la manière dont la subjectivité, en visant à appréhender la réalité, en dévoile justement le caractère fictionnel. Pour paraphraser Adorno (2003, p. 76-77), on pourrait dire que L'entretien du désespoir, à l'instar de tout essai, interroge la "non-vérité" de la vérité. C'est pour cette raison que la démarche de la subjectivité

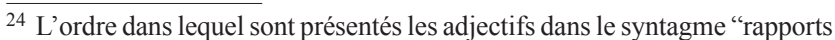
économiques et sociaux" évoque on ne peut mieux les renversements qui sont le noud de la problématique de cet essai de Lapierre.
}

essayistique se heurte inévitablement à la doxa (cf. VIGNEAULT, 2004) et que l'on peut, avec Adorno, la comparer à "l'hérésie" (2003, p. 84).

Si la subjectivité essayistique réussit donc à démasquer la fictionnalité de la réalité, n'oublions pas que la quête de sens qui l'anime ne saurait s'achever. Comment en pourrait-il être autrement? Montaigne ne soutenait-il pas que 1" "agitation et la mutation des pensées" qu'il éprouvait à titre essayiste sont par principe "continuelles" (1998, p. 249)?

De fait, face au vécu fuyant qu'elle s'efforce éperdument de saisir, la subjectivité essayistique, guidée par son intuition, est en quelque sorte obsédée, hantée par des idées et des sentiments auxquels elle cherche une signification, un sens, une expression. Tout porte alors à croire qu'elle est vouée à une sorte de sém(h) antisme, terme par lequel nous désignons cette condition de la subjectivité essayistique où elle se voit tiraillée entre sa sensibilité particulière d'appréhender les essences du vécu et son incapacité à les faire passer de manière limpide, uni-voque, au domaine langagier. Ainsi hantée par les essences, elle hante, pour sa part, les aires sémantiques du langage afin de dire la non-vérité de la vérité, l'irréel du réel. Or, pour acharné que soit son effort, elle ne peut esquisser que les contours des dynamiques essentielles qu'elle croit saisir, elle ne peut que les é-voquer par consonances, assonances et dissonances, pour ensuite, à la manière du poète, laisser libre cours à l'imagination du lecteur qui finira par en saisir les profondes résonnances. Il y a donc fort à parier que le sém(h)antisme de la subjectivité essayistique est à la source de l'aspect poétique de l'essai.

Tendons alors l'oreille au passage suivant qui é-voque sur un mode lyrique la quête sém(h)antique:

N'y a-t-il pas quelque chose au-delà de cet entretien? Quelque chose d'irrécupérable en termes spectaculaires, un non-spectacle œuvrant à même le manque, et perdant immédiatement de ce fait toute exemplarité? (ED, p. 59)

Quelque chose d'autre, irrecevablement: contre l'entretien du désespoir, une joie sans nom, justement étrangère à toute propriété, indemne de toute aliénation. Peut-être silencieuse. On l'imagine facilement ainsi. Mais plus encore que silencieuse elle serait libre, gratuite: offrande ignorante de ce qu'elle offre, dont la grâce impossédable ne peut être donnée. ( $E D$, p. 59)

$J e$ ne sais pas ce que je sais. Et si j'écris c'est oublieusement. Seule condition, la lenteur. Entrer dans la lenteur. C'est là le plus difficile, accepter la lenteur, la laisser devenir ce lieu où la vie ne s'étourdit plus d'une impatience folle, où le désir ne se précipite pas aussitôt, dans une frénétique seconde, vers le regret. Beauté morte de beauté, morte de hâte, morte de peur $[\ldots] .(E D$, p. $60-$ nous soulignons $)$ 
Qu'elle soit si lente, que précisément elle n'est de cesse, ne sache ni s'interrompre ni conclure, on ne suppose pas. L'inexprimable, l'irrécupérable: joie sans nom, joie sans nous [...]. (ED, p.61)

Plusieurs remarques s'imposent au sujet de ce beau passage. D'abord, c'est ici que la subjectivité essayistique apparaît le plus explicitement puisque le pronom "je" qui s'y accuse ne renvoie pas cette fois-ci à un "je" universel, mais à la subjectivité essayistique même. Et qu'avouet-elle à cette singulière occasion? Rien d'autre que ce qu'avoue le "moi" de Montaigne par le fameux "Que sais-je?" des Essais: la véritable subjectivité essayistique part à la recherche de réponses sans savoir où sa quête la mène. Toujours est-il que, grâce à son appréhension intime du vécu, elle ressent "l'inexprimable", "l'irrécupérable", en l'occurrence, la joie sans nom. Bien que cette joie demeure "inexprimable", la subjectivité, en ayant recours à l'expression lyrique, parvient à en é-voquer la nature et la tonalité, lesquelles retentiront différemment dans l'être intime de chaque lecteur.

En effet, les associations variées que nous avons pu observer entre les fragments textuels qui composent $L$ 'entretien $d u$ désespoir ne sauraient s'activer sans interpeller l'imaginaire du lecteur puisqu'il incombe à celui-ci d'en déceler les sens et les significations. Ainsi, le lecteur participe-t-il pleinement au dialogue qui s'établit dans L'entretien $d u$ désespoir et qui se nourrit autant de l'interaction entre la subjectivité essayistique et les divers acteurs de la scène énonciative, que des "éclats" multicolores et multisonores qui unissent les fragments de réflexion de ce véritable essai.

\section{Conclusion}

Témoignant d'un grand nombre d'énonciateurs et de différents rapports qu'entretient la subjectivité essayistique avec eux, L'entretien du désespoir de René Lapierre illustre avec brio que l'essai repose, plus que ne le font le roman ou la nouvelle, sur un acte de "coénonciation ${ }^{25}$ ". Mais qui dit co-énonciation, dit aussitôt co-opération et surtout conscience de l'Autre, donne qui influe à tous égards sur la démarche discursive propre à l'essai.

Fragmentaire et dialogique, cette démarche vise à dessiller les yeux de quiconque désire appréhender l'artificialité de la réalité et saisir le vécu à un niveau essentiel. Elle avance par associations plus ou moins inopinées, plus ou moins significatives, plus ou moins assurées, faisant appel aux ressources du lyrisme qui a bien compris que le monde signifiant ne s'offre pas à la compréhension humaine sans résistances, sans hésitations, sans incertitudes. Autant dire que le lyrisme, quelques vastes ressources langagières qu'il possède, ne sait pas résoudre le sém(h)antisme de la subjectivité essayistique; tout au plus peut-il l'aider à l'atténuer en lui fournissant des outils nécessaires pour esquisser les contours des essences existentielles qu'elle a entrevues, et pour prolonger et partager les questionnements fondamentaux qui la rongent. Ici, L'entretien du désespoir s'avère une dernière fois exemplaire, dans la mesure où les fragments de réflexion de type "éclats" qui constituent cet essai rejaillissent sur le lecteur bienveillant de telle sorte qu'il ne peut qu'entrer en dialogue avec l'univers mouvementé qui s'y déploie. Il risque même d'être si emporté par les interrogations qui traversent cet univers que sa perception de la réalité changera et que la prochaine fois qu'il lira une annonce publicitaire affichant un slogan du genre "12 jours: 12 façons de dire je t'aime ${ }^{26 ", ~ l e ~ d i a l o g u e ~}$ déclenché chez lui par $L$ 'entretien du désespoir résonnera de nouveau dans les profondeurs de son âme.

C'est somme toute sous le signe du partage et du dialogue qu'il faut inscrire l'œuvre entière de la subjectivité essayistique et évaluer le succès de sa démarche. Car son œuvre aura atteint sa finalité moins en apportant des réponses précises et définitives aux questions délicates qu'elle pose à propos des vérités toutes faites qu'en continuant, au-delà de la dernière page de l'essai, l'échange dialogique amorcé autant avec ellemême qu'avec des interlocuteurs variés.

$\mathrm{Ne}$ serait-ce pas, en dernière instance, dans cette méfiance à l'égard de la doxologie, dans cette suspicion vis-à-vis de réponses uniques et unidimensionnelles, et dans cette ouverture foncière aux échanges avec l'Autre, qu'il faudrait chercher l'attrait qu'exercice la pratique de l'essai auprès des penseurs américains? D'autant qu'ils tendent souvent, comme Montaigne, à savoir ce qu'ils fuient, mais non pas ce qu'ils cherchent.

\section{Références}

ADORNO, Theodor. L'essai comme forme. Trad. Sibylle Muller. In: François Dumont (Ed.). Approches de l'essai: anthologie. Québec: Nota bene, 2003 [1958]. p. 49-84.

ANGENOT, Marc. La parole pamphlétaire: contribution à la typologie des discours modernes. Paris: Payot, 1982.

ARISTOTE. Premiers Analytiques. Michel Crubellier (Ed.). Paris: Flammarion, 2014.

AUDET, René. Tectonique essayistique: raconter le lieu dans l'essai contemporain. Études littéraires, v. 37, n. 1, p. 119-131, aut. 2005.

BELLEAU, André. Surprendre les voix. Montréal: Éditions du Boréal, 1986.

\footnotetext{
${ }^{25}$ Le terme est de Vandendorpe. Il l'emploie pour rendre compte de la nature de tout acte de lecture (2004, p. 137).

${ }^{26}$ Annonce publicitaire de la compagnie de la Baie Hudson parue en décembre 2006
} 
D'ALEMBERT, Jean le Rond. Essai. In: DIDEROT, Denis; Jean le Rond d'ALEMBERT (Eds.). Encyclopédie, ou Dictionnaire Raisonné des Sciences, des Arts et des Métiers, $1^{\text {re éd. }}$ [1751]. Base textuelle "Encyclopédie Diderot et d'Alembert" (Projet ARTFL). Disponible en ligne: <http://portail.atilf.fr/ encyclopedie/index.htm>. Site consulté le 24 avril 2015.

DION, Robert; Anne-Marie CLÉMENT; Simon FOURNIER. Les "essais littéraires" aux Éditions de l'Hexagone (19881993): radioscopie d'une collection. Québec: Nota bene, 2000.

FOX, Claire F. Commentary: The Transnational Turn and the Hemispheric Return. American Literary History, v. 18, n. 3, 2006, p. 638-647.

LA BAIE. 12 jours: 12 façons de dire je t'aime (Publicité, Canada). Déc. 2006.

GENETTE, Gérard. Figures III. Paris: Seuil, 1972.

IMBERT, Patrick. Comparer le Canada et les Amériques: Des racines aux réseaux transculturels. Québec: PUL, 2014.

ISSENHUTH, Jean-Pierre. Rêveries. Montréal: Boréal, 2001 .

KERBRAT-ORECCHIONI, Catherine. L'énonciation de la subjectivité dans le langage. Paris: Armand Colin, 1980.

LAPIERRE, René. Aimée soit la honte. Montréal: Les Herbes rouges, 2010.

LAPIERRE, René. Comme des mannequins. Montréal: Primeur, 1983.

LAPIERRE, René. Écrire l'Amérique. Montréal: Les Herbes rouges, 1995.

LAPIERRE, René. Effacement. Montréal: Éditions de l'Hexagone, 1991.

LAPIERRE, René. Fais-moi mal Sarah. Montréal: Les Herbes rouges, 1996.

LAPIERRE, René. Figures de l'abandon. Montréal: Les Herbes rouges, 2002.

LAPIERRE, René. Là-bas c'est déjà demain. Montréal: Les Herbes rouges, 1994.

LAPIERRE, René. La carte des feux. Montréal: Éditions Les Herbes rouges, 2015.

LAPIERRE, René. L'atelier vide. Montréal: Les Herbes rouges, 2003.

LAPIERRE, René. L'eau de Kiev. Montréal: Les Herbes rouges, 2006.

LAPIERRE, René. L'entretien $d u$ désespoir: essai sur l'affolement. Montréal: Les Herbes rouges, 2001.

LAPIERRE, René. Les masques du récit. Québec: Hurtubise HMH, 1980.

LAPIERRE, René. L'Été Rebecca. Paris: Seuil, 1985.

LAPIERRE, René. L'imaginaire captif. Montréal: Les Quinze/ Éditions de l'Hexagone, 1981/1992.

LAPIERRE, René. Love and Sorrow. Montréal: Les Herbes rouges, 1998.

LAPIERRE, René. Piano. Montréal: Les Herbes rouges, 2001.

LAPIERRE, René. Pour les désespérés seulement. Montréal: Les Herbes rouges, 2012
LAPIERRE, René. Profil de l'ombre. Trois-Rivières: Les Écrits de forges, 1983.

LAPIERRE, René. Traité de physique. Montréal: Les Herbes rouges, 2008.

LAPIERRE, René. Viendras-tu avec moi? Montréal: Les Herbes rouges, 1996.

LAPIERRE, René. Une encre sépia. Montréal: Éditions de l'Hexagone, 1990.

LUKÁCS, Georg. L'âme et les formes. Trad. Guy Haarscher. Paris: Gallimard, 1974 [1911].

MAILHOT, Laurent. L'essai québécois depuis 1845: étude et anthologie. Montréal: Hurtubise HMH, 2005.

MARCEL, Jean. Pensées, passions et proses. Montréal: L'Hexagone, 1992.

MONTAIGNE, Michel de. Essais (Livre II). Pierre Villey (Ed.). Paris: PUF, 1924 [1580].

MONTAIGNE, Michel de. Essais (Livre III). André Tournon (Ed.). Paris: Imprimerie nationale, 1998 [1580].

NARDOUT-LAFARGE, Élisabeth. L'émergence de la voix essayistique dans les textes de Monique Bosco. In: Anne Caumartin et Martine-Emmanuelle Lapointe (Eds.). Parcours de l'essai québécois (1980-2000). Québec: Nota bene, 2004. p. 31-45.

ORTEGA Y GASSET. Obras compl. (Tome I). [S.1.]: [S.é.], 1936.

OUELlET, Pierre. Le premier venu. Poétique du passant. Montréal: Éditions du Noroît, 2003.

SARMIENTO, Domingo Faustino. Facundo. Paris: Stock, 1934 [1845].

SIEMERLING, Winfried. Récits nord-américains d'émergence: culture, écriture et politique de re/connaissance. Trad. Patricia Godbout. Québec: PUL, 2010 [2005].

TERRASSE, Jean. Rhétorique de l'essai littéraire. Québec: Les Presses de l'Université du Québec, 1977.

TREMBLAY, Yolaine. Du Refus global à la responsabilité entière. Parcours analytique de l'essai québécois depuis 1948. Sainte-Foy: Le Griffon d'argile, 2000.

VANDENDORPE, Christian. Du fragmentaire et de la subjectivité dans l'essai. In: Anne Caumartin et MartineEmmanuelle Lapointe (Eds.). Parcours de l'essai québécois (1980-2000). Québec: Nota bene, 2004. p. 127-144.

VIGNEAULT, Robert. L'écriture de l'essai. Montréal: L'Hexagone, 1994.

VIGNEAULT, Robert. Notre sauvage besoin de libération. In: Anne Caumartin et Martine-Emmanuelle Lapointe (Eds.). Parcours de l'essai québécois (1980-2000). Québec: Nota bene, 2004. p. 17-30.

WALTER, Roland. Narrative Identities: (Inter)Cultural InBetweenness in the Americas. Berne: Peter Lang, 2003.

WHITMORE, Charles E. The Field of the Essay. PMLA, v. 36, n. 4, p. 551-564, 1921.

Recebido: 25 de abril de 2015

Aprovado: 15 de julho de 2015

Contato: emir.delic@usainteanne.ca 